\title{
Dificultades y estrategias en el manejo del régimen terapéutico en el paciente renal crónico en hemodiálisis
}

\author{
António Filipe Cristóvão
}

Mestrado en ciencias de enfermagem. Profesor Escola Superior de Enfermagem de Lisboa

\section{Resumen}

Objetivo: identificar las dificultades de los pacientes de hemodiálisis en el manejo del régimen terapéutico, las estrategias para hacer frente a estas dificultades e identificar factores que influyen en la gestión del tratamiento.

Pacientes y métodos: se realizaron entrevistas semiestructuradas a una muestra intencional de 20 pacientes adultos, de dos centros de hemodiálisis, sometidas a análisis de contenido temático. Resultados: la mayor parte de las dificultades es en el área psicosocial: restricción de líquidos; dieta restrictiva; tener que realizar hemodiálisis; duración del tratamiento; tomar la medicación; limitaciones de vacaciones. Para hacer frente a las dificultades, los participantes utilizan más las estrategias de resolución de problemas que las estrategias para el control de las emociones.

Conclusiones: el equipo de salud, la familia y la función renal residual, facilitan el manejo del tratamiento, mientras que el calor del verano y el humo son factores de complicación. Las enfermeras han de entender cómo los pacientes controlan el régimen de tratamiento, para promover mejor su autonomía.

Correspondencia:

António Filipe Cristóvão

Escola Superior de Enfermagem de Lisboa

Dpto. Enfermagem Médico-cirúrgica

Avda. Prof. Egas Moniz

1600-190 Lisboa. Portugal

E-mail:acristovao@esel.pt
PALABRAS CLAVE:

- ADAPTACIÓN

- FALLO RENAL CRÓNICO

- HEMODIÁLISIS

Difficulties and strategies to manage the therapeutic regimen in the patients with chronic renal in hemodialysis

\section{Abstract}

Objective: to identify the difficulties perceived by hemodialysis patients related to the management of the therapeutic regimen; the strategies they use to cope with these problems, and identify factors that influence the management of the treatment.

Methods: we conducted semi structured interviews 20 adults from two hemodialysis centers and thematic content analysis.

Results: most difficulties are identified as psychosocial: fluid restriction; dietary restriction; having to perform hemodialysis; ${ }_{\text {; }}$ duration of hemodialysis sessions; taking prescribed medication; limitations on vacancy. Subjects mobilize different strategies to cope with difficulties, but problem-focused strategies are more used than emotion-focused strategies.

Conclusions: support from the health care team, from family and having residual renal function, seem to facilitate treatment management, while the summer heat and smoking are among the factors hindering. Nurses need to understand how renal patients manage the treatment regimen, so they can better help and promote their independence. 
KEY WORDS:

- COPING

- KIDNEY FAILURE CHRONIC

- HEMODIALYSIS

\section{Introducción}

La enfermedad renal crónica (ERC) es un problema de salud en todo el mundo y corresponde a una pérdida progresiva e irreversible de la función renal, que puede progresar a la necesidad de terapia de reemplazo renal (TRR). Según la Sociedad Portuguesa de Nefrología (SPN), Portugal registró a finales de 2012 una prevalencia de pacientes en TSR $1.670,02$ pmp $^{(1)}$. En el estadio 5 de la ERC, la mayoría de los pacientes deben cumplir con un programa de hemodiálisis (HD), cuidar de su acceso vascular, cumplir con las restricciones de agua y dietéticas, y tomar varios medicamentos. Los pacientes en HD son sometidos a altos niveles de estrés debido a la restricción dietética y líquidos, la fatiga, la incertidumbre sobre el futuro, los calambres, las limitaciones en la actividad física y social, la pérdida de ingresos y los cambios en los roles ${ }^{(2-8)}$. Para hacer frente a estas adversidades los pacientes en HD movilizan estrategias de adaptación, Las estrategias destinadas a la resolución de problemas son más comunes y más eficaces que las del control emocional(3,5) $(9-10)$. El autocuidado es una función humana que los individuos juegan deliberadamente para preservar la vida, la salud, el desarrollo y bienestar ${ }^{(11)}$, pero tiene que ser aprendido y aplicado de acuerdo con las necesidades de salud de la persona ${ }^{(12)}$. La capacidad del paciente para el cuidado personal y para administrar el régimen de tratamiento depende del estado de salud, la capacidad cognitiva y de los recursos familiares y sociales. La ineficacia de la gestión del tratamiento se asocia con regímenes de medicamentos complejos, la falta de conocimiento, falta de habilidades para llevar a cabo el tratamiento y los resultados positivos del tratamiento ${ }^{(13)}$. Todos estos argumentos se aplican a los pacientes renales en HD. Como las intervenciones educativas no garantizan un autocuidado duradero y el autocuidado es fundamental para promover la buena gestión del régimen terapéutico, se hacen necesarios más estudios para la comprensión de la experiencia de los pacientes con enfermedad renal crónica en hemodiálisis en la gestión del régimen terapéutico.

Para comprender la experiencia de los pacientes en el autocuidado, se desarrolló un estudio con los siguientes objetivos: identificar las dificultades percibidas por los pacientes en la gestión del régimen terapéutico relacionados con la hemodiálisis renal crónica, identificar estrategias de adaptación, usadas por los pacientes para hacer frente a estas dificultades e identificar los factores que facilitan o dificultan la gestión de régimen terapéutico.

\section{Pacientes y métodos}

Se utilizó una metodología cualitativa mediante el método exploratorio con entrevistas semiestructuradas.

La muestra fue intencional y estuvo compuesta por 20 pacientes con enfermedad renal crónica mayores de 18 años, con al menos 6 meses en programa regular de $H D$, en tratamiento en un centro extra hospitalario de diálisis en Lisboa, y que fueran capaces de describir su experiencia en la gestión de régimen terapéutico. El estudio fue aprobado por el Comité de Ética de la institución y los participantes firmaron un consentimiento informado. Las entrevistas semiestructuradas se realizaron en un ambiente tranquilo y reservado de la unidad de diálisis. Las entrevistas con una duración aproximada de $35 \mathrm{~min}$, se llevaron a cabo antes del inicio de las sesiones de HD, fueron audio grabadas, transcritas, y sometidas a análisis de contenido temático. Las declaraciones permitieron encontrar algunas de las categorías previamente identificadas en la revisión de la literatura, pero nuevas categorías surgieron acerca de cómo los pacientes renales en HD controlan el régimen de tratamiento. Después de una primera lectura de las entrevistas y establecido el análisis de corpus, codificamos las unidades de registro, de las que surgieron los temas y categorías. Las categorías se definen dentro de las normas de uniformidad, integridad, exclusividad, objetividad y pertinencia. Dos investigadores con experiencia en el análisis de contenido, clasificaron una muestra de dos entrevistas. La comparación de los resultados reveló un amplio consenso, por lo que las categorías fueron aceptadas como válidas.

\section{Resultados}

La edad promedio de los participantes fue de $57,5 \pm$ 15,4 años, entre 28 y 81 años. La mayoría (12, 60\%) fueron hombres y el tiempo promedio de permanencia en el programa regular de HD fue de 4,13 años. Las principales condiciones comórbidas fueron la diabetes ${ }^{(2)}$ y cálculos renales ${ }^{(1)}$. Mediante el análisis de contenido 
se identificaron tres temas: dificultades en el manejo del régimen terapéutico, las estrategias para hacer frente a las dificultades y los factores que influyen en la gestión del tratamiento.

a) Las dificultades en la gestión del régimen terapéutico. Este tema incluye dos categorías: las dificultades psicosociales asociadas a situaciones evaluadas como una amenaza al bienestar, 0 relacionadas con la disfunción social, con predominio de componente afectivo y síntomas psicológicos; y las dificultades fisiológicas relacionadas con las perturbaciones de los sistemas y tejidos del cuerpo, que se manifiestan por síntomas físicos (tabla 1).

\begin{tabular}{|c|c|}
\hline Categorías & Subcategorías ( $\mathrm{n}^{0}$ de participantes) \\
\hline Dificultades Psicosociales & $\begin{array}{l}\text { Restricción de líquidos (15) } \\
\text { Restricción dietética (10) } \\
\text { Obligación de hemodiálisis (9) } \\
\text { Duración de la hemodiálisis (9) } \\
\text { Tomar a medicación como prescrita (8) } \\
\text { Limitación en relación a vacaciones (5) } \\
\text { No poder llevar a cabo actividades so- } \\
\text { ciales (3) } \\
\text { Conciliar la hemodiálisis con el trabajo (2) } \\
\text { Enfermera indiferencia (1) } \\
\text { El tiempo empleado en el transporte (1) } \\
\text { El miedo a perder el acceso vascular (1) }\end{array}$ \\
\hline Dificultades Fisiológicas & $\begin{array}{l}\text { Dolor à la punción (4) } \\
\text { Complicaciones intradialíticas (3) } \\
\text { Dolor en el acceso vascular (1) } \\
\text { El dolor después de la diálisis (1) }\end{array}$ \\
\hline
\end{tabular}

Tabla 1. Dificultades relacionadas con la gestión del régimen terapéutico.

En la subcategoría restricción de líquidos, 15 participantes encontraron doloroso no poder beber líquidos en la cantidad deseada, declarando: "La sed es peor." (S3); "No beber agua, o la prohibición de tomar líquidos, es la parte difícil"(S4); "la restricción de líquidos es más complicado, ya que el tratamiento causa sed." (S10); es muy difícil controlar la sed." (E17); Las restricciones en la subcategoría dieta (10 participantes) muestra la incomodidad de no poder comer algunos alimentos con la frecuencia 0 la cantidad deseada: "Me quitaron estas comidas, que no son convenientes porque los granos y cereales tienen mucho fósforo, ya que la leche, más allá de neta tiene mucho fósforo." (S9); "Tener que comer fruta cocida y no poder comer sopa es una cosa que hace una gran diferencia." (S18). La obligación de hemodiálisis causa molestias al suponer continuar el tratamiento indefinidamente, lo que se percibe en las siguientes frases: "Es como mandarme a la horca.
Prefiero estar en la cárcel que venir aquí. Después de un tiempo habría cumplido con la cárcel..." (S8). La subcategoría Duración de las sesiones de hemodiálisis revela el malestar del paciente, limitado a un espacio pequeño, con la obligación de cumplir con el tiempo completo del tratamiento, y no siendo capaz de hacer otras actividades. Los participantes dijeron: "Son cuatro horas que me retiro de otras actividades." (S5); "Otro aspecto está relacionado con el momento de la detención, entre comillas, que es el tiempo en diálisis." (S12).

Tomar la medicación según la prescripción es un problema asociado a la polifarmacia, la pérdida de la memoria, la atención y la visión (sobre todo en los ancianos), o ardor de estómago causado por ciertos medicamentos. Los pacientes dijeron: "Es muy complicado, hay quince pastillas al día." (S3); "A veces es difícil porque se me olvida". (S11); "(...) así que tomó dos pastillas iba a vomitar." (S13). En la subcategoría limitaciones en relación a las vacaciones destaca la burocracia y la falta de lugares para realizar HD en el momento previsto. Los participantes informaron: "Necesito credenciales. Se trata de una burocracia." (S12); "No siempre podemos ir de vacaciones a donde queremos, porque no existe un centro de tratamiento cercano o no hay vacantes en la zona." (S16). La subcategoría no poder llevar a cabo actividades sociales significa no poder viajar, el acceso a familiares y amigos, el estudio y la diversión. Uno de los participantes dijo: "El tiempo perdido en la diálisis me impide estudiar o hacer otro tipo de actividades." (S10). La subcategoría conciliar la hemodiálisis con el trabajo muestra la dificultad de conciliar los programas de tratamiento y el trabajo. Se concluye con las expresiones: "Es muy difícil conciliar el tratamiento y la vida profesional y familiar." (S6). Entre las dificultades fisiológicas resalta el dolor a la punción asociado a la variación del punto de inserción de la aguja: "Ni siquiera puedo mirar. Parecen ganchillos, me impresiona." (S8). La subcategoría complicaciones intradialíticas muestra que en las sesiones se siguen produciendo cambios de tensión, calambres, náuseas y vómitos. Los pacientes reportaron: "La tensión Ilegó a 200/100." (S5); "El martes me cansé de vomitar."(E8).

b) Estrategias para abordar las dificultades en la gestión de régimen terapéutico: Este tema reúne los esfuerzos cognitivos y de comportamiento para hacer frente a los problemas relacionados con el 
tratamiento. Se identificaron dos categorías: las estrategias centradas en la resolución de problemas, de modificación de la situación, y las estrategias de control de las emociones, para disminuir la presión emocional $^{(9)}$.

Para hacer frente a la restricción de agua los pacientes utilizan 17 estrategias. La mayoría (15) estaba orientada a la solución de problemas: la reducción del volumen de fluido ingerido (14 pacientes); se esfuerzan para no beber (9); evaluar la diuresis (8); limitar el volumen de ingesta de agua a una botella (8); controlar el aumento de peso interdiálisis (7); chupar o masticar hielo (5); controlar la ingesta de líquidos (4); beber líquidos fríos (4); evitar alimentos dulces y salados (4); gárgaras (4); el hacer sopa espesa (3); beber poco cada vez (3), beber agua caliente (2); beber solo con las comidas (1), y beber alcohol sólo en los días de fiesta (1). También presentaran dos estrategias para el control de las emociones: Mantenerse ocupado (1), y minimizar el problema (1); El discurso de los participantes destacamos: "Bebo leche en una taza de café." (S8); "Mido la orina [diuresis] por lo general en el fin de la semana."(E8); "Llevo una botella pequeña y sé que no puedo beber más de una botella..."(S10); "Hago todo lo posible para no venir aquí con más de dos kilos."(S5); "Cuando tengo sed como un cubo de hielo." (S10); "(...) enjuague con agua tibia." (S2); "No como cosas saladas."(E15), "La sopa se debe comer con un tenedor..."(E3); "Si estoy siempre ocupado o fuera de casa, yo no bebo." (S4).

Para hacer frente a las restricciones dietéticas los pacientes no sólo utilizan estrategias centradas en el control emocional.

- Aceptan las restricciones (1 paciente), y presentan 10 estrategias centradas en la resolución de problemas: consumo moderado de ciertos alimentos (15), evitando ciertos alimentos (10), la cocción de alimentos (6); poco comer (5), reducir la sal (5), cumplir con el rigor la dieta (4), la búsqueda de información (4), fraccionar las comidas (2), comer inmediatamente antes de la diálisis (2), y el control de la ganancia de peso interdiálisis (1). Se destacan las expresiones: "No como mucho vegetal, ya que tiene mucho potasio."(S2); "Me gusta un poco de todo sin abusar." (S12); "Cómo siempre la parte más pequeña."(S13); "Si no es para comer, no como!" (S9); "Cómo muy poco desde que hago diálisis." (S2); "Si almuerzo, no voy a cenar!"(S9); "Hervir las verduras en agua y después desechar el agua. Es una molestia!"(S8); "Cómo todo sin sal."(E13); "Trato de comer cosas con más potasio en los días de diálisis."(S18).

Los participantes indicaron 9 estrategias para satisfacer la necesidad de tener la hemodiálisis. Ocho están apuntando el control de las emociones: aceptar la realidad (12 pacientes), la esperanza en el trasplante (4), asignar un significado positivo a la diálisis (4), considerar el tratamiento como un trabajo (3), no pensar en él (3), distraerte (2), pensar en algo agradable (1) y orar. Sólo uno eligió una estrategia de resolución de problemas: buscar información (1). Destacamos las siguientes declaraciones: "Tengo que aceptar las cosas como son, no puede ser de otra manera." (SI); "EI objetivo es que esperar para un trasplante." (S14), "Hemos venido aquí para conseguir un poco de nuestra salud."(S15); "Esto es un part-time que no te puedes perder." (S13).

Para hacer frente a la duración de las sesiones de hemodiálisis los participantes revelaron nueve estrategias. Cinco de ellas orientadas al control emocional: lectura (4 pacientes), ver películas 0 programas de televisión (3), hablar (2), tratando de dormir (2), y escuchar música (1). Las cuatro estrategias específicas para resolver problemas fueron: solicitud para reducir la duración del tratamiento (1), acudir al médico (1), reconocer la necesidad de tratamiento (1), y pedir la interrupción del tratamiento (1). El reporte de los pacientes incluye: "Escucho música y me abstraigo." (S6); "Veo una película y se pasan dos horas." (16); "Si usted quiere vivir un poco más debe tener la hemodiálisis." (S14); "Trato de dormir". (S16); "He hablado con el médico para reducir a dos veces [sesiones de diálisis] por semana o reducir el tiempo para tres horas y media."(S19); "Hay días que pido que me desconecten para ir al baño". (S19).

En cuanto a la dificultad de tomar la medicación prescrita los pacientes indicaron 10 estrategias, incluyendo 7 de resolución de problemas: crear una rutina (6 pacientes), utilizar un dispensador de pastillas (6), esforzarse por cumplir con el tratamiento (4), llevar sus medicamentos con usted (3); registrar el medicamento prescrito (2), obtener ayuda del equipo de apoyo para la salud (1) obtener ayuda del cónyuge (1). Las tres estrategias de 
control emocional incluyen: asignar un significado positivo para el tratamiento (1), restar importancia (1), y aceptar la realidad (1). Las declaraciones de los pacientes muestran estas estrategias: "Tengo una caja con separadores y los pongo siempre en la mañana." (S16); "(...) siempre hay que tener atención... (S13); "Hablé con el doctor, me tomaba cuatro y ahora tomo sólo dos. (E2).

Para hacer frente a las limitaciones en relación a las vacaciones, los pacientes reportaron el uso de tres estrategias. Dos eran orientados a la resolución de problemas: la planificación del tratamiento en vacaciones (4 pacientes), y falta de una sesión de diálisis (2). La estrategia emocional era el cumplimiento (1). Ese es el significado de frases como: "Si me voy de vacaciones tengo que preparar todo antes." (S4); "Saltar la diálisis el miércoles, que es menos peligroso. Después de la sesión de lunes para venir la noche del viernes. Tengo vacaciones de cuatro días."(E16); "He resuelto el problema. Mira, no me he ido de vacaciones!"(S7).

Para lidiar con el dolor de la punción venosa, los pacientes utilizan ambos tipos de estrategias, pero predominan en las estrategias de control emocional: evitar pensar en él (1), evitar mirar (1), aceptar la situación (1), y relajarse (1). Las estrategias centradas en la solución de problemas incluyen: la aplicación de anestesia tópica (2), y pregunte por golpear en el mismo lugar (1). Es lo que se percibe de las siguientes afirmaciones: "Me puse la pomada y es anestesiado y no duele nada." (S8); "Lo que tiene del callo y la entrada de la aguja es más fácil." (S3); "Trato de olvidar, no vale la pena pensar." (S8); "Debe ser así. Si no lo hago, mal para mí." (S20); "Me relajo y creo que tengo que ser fuerte como los otros."(S20).

c) Factores que influyen en el régimen terapéutico. Este tema incluye dos categorías: factores facilitadores y factores que dificultan el manejo del tratamiento (Tabla 2). Entre los factores de riesgo destacar la subcategoría apoyo de los cuidadores ( 15 pacientes). Los médicos ayudan en la prescripción del tratamiento, en la dieta y la medicación, los dietistas ayudan a controlar su dieta y fluidos; las enfermeras ayudan a resolver complicaciones intradialíticas y ajustan el tiempo de diálisis. Las siguientes declaraciones ilustran esta subcategoría: "El doctor dice que tenga cuidado con el fosforo." (S15); "La dietista me dio un libro para ver los alimentos que puedo comer. (S4); y "Las enfermeras también nos aconsejan en ungüentos y esto pasa."(E3).

\begin{tabular}{l|l}
\hline Categorías & Subcategorías ( ${ }^{0}$ de participantes) \\
\hline Soporte de profesionales de la salud (15) \\
El apoyo familiar (14) \\
El mantenimiento de la función renal re- \\
sidual (9) \\
Características de la personalidad (9) \\
La actitud del equipo de atención de la \\
salud (6) \\
El apoyo de los pacientes y amigos (6) \\
Tener una ocupación (3) \\
Estar en la lista de espera de trasplante (3) \\
Asociaciones de apoyo (2) \\
Capacidad de los enfermeros (2) \\
Disponer de medios de distracción (1) \\
Estar acostumbrado al aumento de ingesta \\
de agua (4) \\
El calor del verano (3) \\
Tener que comer fuera en el restaurante (1) \\
Fumar (1) \\
Cambio de rutinas en la sala de diálisis (1) \\
Ruido (1) \\
Los conflictos en la sala de diálisis (1) \\
Burocracia (1)
\end{tabular}

Tabla 2. Los factores que influyen en la gestión del régimen terapéutico.

El apoyo familiar (14 pacientes) destacan el apoyo de la pareja y la familia. Los padres ofrecen apoyo psicológico, económico y de transporte: la esposa y la hermana ayudan en la alimentación y los medicamentos, y el marido ofrece ayuda psicológica, económica y en el desplazamiento. Las siguientes expresiones traducen esta subcategoría: "Mi esposa se encarga de la preparación de la medicación todos los días." (S1); "Mi marido viene a recogerme y tiene paciencia porque esta enfermedad no es fácil."(S2); "Mi padre lleva e recoge en el coche porque se retiró recientemente." (S10). Mantener la función renal (9 participantes) facilita la gestión de la restricción de líquidos. Este es el significado de las declaraciones atribuidas: "Orino suficiente, lo que es una gran cosa!" (S2); "Si no orino debe ser más complicado, ya que puedo estar hinchada." (S14). La subcategoría características de la personalidad (9 participantes) incluye optimismo, determinación y capacidad de adaptación, las condiciones que permitan enfrentar mejor los problemas y se resisten a la adversidad. Estas son algunas de las frases: "Vengo aquí y me olvido de todo. Soy muy juguetón." (S15); "(...) fuerza de voluntad es lo que hace que me encargue de todas estas cosas." (S13). La subcategoría la actitud del equipo de salud aprecia la voluntad de responder 
a las quejas de los pacientes, con la participación en las decisiones y la preocupación por su salud. Citamos algunas declaraciones: "Es muy importante la atención, la disponibilidad y la comprensión de los profesionales. Son situaciones en las que las personas son vulnerables y los detalles marcan la diferencia."(S6); "Llegué a casa y la hora de la cena, el médico de la clínica me llamó para preguntarme cómo estaba. (S9). Entre los factores que complican la gestión del régimen terapéutico pone de relieve la subcategoría estar acostumbrado a beber más agua. En las etapas anteriores de la enfermedad renal los pacientes se orientan al refuerzo de la ingesta de agua, y les cuesta mucho ahora tener que reducir su consumo. Es el significado de los siguientes registros: "Antes de comenzar la hemodiálisis bebí mucha agua siguiendo el consejo médico y porque me gustaba." (S7); "Bebí de tres a cuatro litros de agua por día. Por supuesto, ahora con la hemodiálisis tengo que cambiar radicalmente." (S16).

En subcategoría el calor del verano los participantes declararon: "La sed cuesta mucho, sobre todo en el verano." (S2); "Bebo más durante el verano." (S12). La subcategoría tener que comer fuera revela la dificultad de encontrar un restaurante que confeccione la dieta renal, como se muestra en la siguiente afirmación: "La parte más difícil es comer fuera. Confeccionado no de la manera que estoy acostumbrado." (S5). El subcategoría fumar favorece la xerostomía y dificulta el control de líquidos. La frase siguiente ilustra esta idea: "EI humo tiende a mantener la boca seca."(S12).

\section{Discusión}

La evolución de la ERC conduce a la necesidad de establecer una TRR. Las personas con ERC tienen grandes dificultades para gestionar un régimen prolongado, complejo y doloroso, que se caracteriza por hemodiálisis frecuente, restricciones dietéticas severas y un régimen de medicamentos complejos. Un análisis del contenido temático de 20 encuestados, ha permitido identificar tres temas: dificultades en la gestión del régimen terapéutico, las estrategias utilizadas por los pacientes para hacer frente a las dificultades, y los factores que influyen en la gestión del tratamiento.

La mayoría (11) de las dificultades identificadas es psicosocial, lo que es consistente con otros hallazgos $^{(2-3),(5,8)}$, y muestra que los problemas psicosociales son tan o más importantes que los de orden fisiológico. Es posible que las nuevas tecnologías permitan una diálisis más estable y segura, y que los problemas fisiológicos sean menos frecuentes y mejor tolerados por los pacientes. Restricciones de fluidos y en la dieta se encuentran entre los principales factores de estrés identificados en varios estudios ${ }^{(2-3),(5,8,14)}$. También se consideraron las dificultades para llevar a cabo actividades sociales o de transporte, como el bienestar inquietante ${ }^{(3,4),(8)}$. No saltar la HD, soportar la duración del tratamiento y tomar la medicación siguen siendo problemas importantes. La indiferencia de la enfermera es especialmente importante porque afecta a la relación de ayuda. El tratamiento es doloroso y vivido en alta tensión emocional, pero que es posible que las enfermeras traten de evitar conflictos con los pacientes a través de una relación distante y limitada a los contactos estrictamente necesarios. Sin embargo, parece que los pacientes mejor adaptados al tratamiento tienen una buena relación con el equipo de salud(15). Los ancianos pueden ser especialmente difíciles en la gestión del régimen de tratamiento debido al deterioro cognitivo y necesitan más atención, haciendo hincapié en la necesidad de una referencia de las enfermeras. El miedo a perder el acceso vascular se justifica porque la eficacia de la diálisis depende de un buen acceso y también porque los ancianos y los diabéticos $(27 \%$ de la población) tienen un mayor riesgo de complicaciones vasculares $^{(1,16)}$.

Los pacientes utilizan muchas estrategias para hacer frente a las dificultades relacionadas con la gestión del régimen terapéutico. Estrategias orientadas a la solución de problemas parecen ser más numerosas y, en ciertas situaciones, más utilizadas que las estrategias dirigidas al control de las emociones, lo cual es consistente con estudios anteriores ${ }^{(3-6),(10)}$. El primer tipo de estrategia parece ser más eficaz en el manejo del estrés ${ }^{(5,10)}$, y por ello es posible que los pacientes tengan tendencia a omitir otras estrategias de control emocional como negar la realidad emocional, fantasear o culpar a otros. Los datos también muestran que las personas tienden a combinar los dos tipos de estrategias cuando se enfrentan a un problema. Esto puede significar que no hay estrategias intrínsecamente mejor que otras, y que las estrategias se eligen en función del contexto y la persona ${ }^{(9)}$. Para enfrentar la restricción de líquidos y la dieta, así como el dolor en el acceso vascular, los pacientes utilizan principalmente estrategias de resolución de problemas. Los pacientes parecen buscar activamente soluciones a estas dificultades y se dan cuenta de ejercer un cierto control sobre estas 


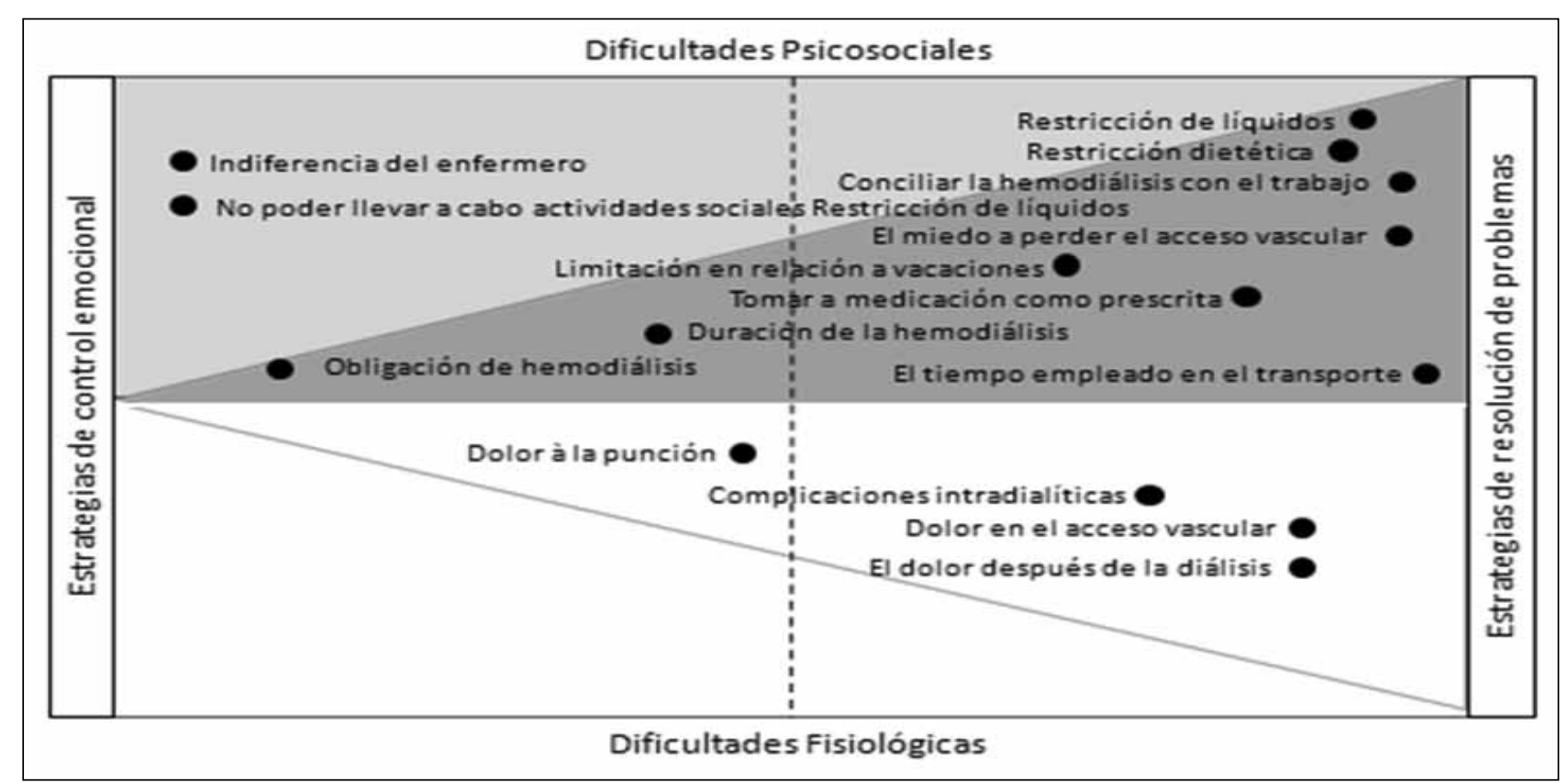

Figura 1. Organización de las dificultades en el tipo de estrategias de adaptación.

situaciones. Si es así, es importante motivar a los pacientes a desarrollar el potencial de hacer frente a esas dificultades. Para otras dificultades (necesidad de hacer la HD, respetar la duración del tratamiento o el dolor a la punción), los pacientes parecen movilizar más estrategias de control emocional, tal vez para reducir el estrés emocional cuando se dan cuenta que no pueden hacer nada para cambiar la situación $\mathrm{n}^{(9)}$. Las enfermeras deben ser conscientes de que el uso de estrategias de evitación, escape o la negación, no agraven la situación de salud de los pacientes.

En la Figura 1 se relaciona los problemas con estrategias de adaptación utilizadas para administrar el tratamiento. Los pacientes parecen controlar mejor las dificultades situados a la derecha de la figura, es decir, la mayoría de las dificultades que enfrentan.

El estudio también identificó los factores que facilitan o dificultan la gestión del régimen terapéutico. Los principales factores facilitadores fueron el apoyo de profesionales de la salud, el apoyo familiar, el mantenimiento de la función renal residual, rasgos de personalidad, actitud del personal de salud, y el apoyo de los pacientes y amigos. Otros factores que se mencionaron menos: conseguir un trabajo, estar en la lista de espera para trasplante renal asociaciones de apoyo, y la habilidad de la enfermera. El médico está muy vinculado a las decisiones sobre la duración de las sesiones de diálisis, medicamentos y las vacaciones, mientras que el dietista se asocia con una mayor educación sobre la dieta y el control de fluidos. Las enfermeras ayudan en el tratamiento de la dieta y en la restricción de líquidos, complicaciones intradialíticas y dolor al punción. Los familiares del género femenino ayudan especialmente en la dieta, los medicamentos y fomentando el tratamiento. Los hombres parecen estar más involucrados en la solución de las dificultades económicas y de transporte. Mantener la función renal residual facilita la gestión de la restricción de líquidos. La Figura 2 ilustra la relación de estos tres factores para los pacientes con dificultades en la gestión del tratamiento.

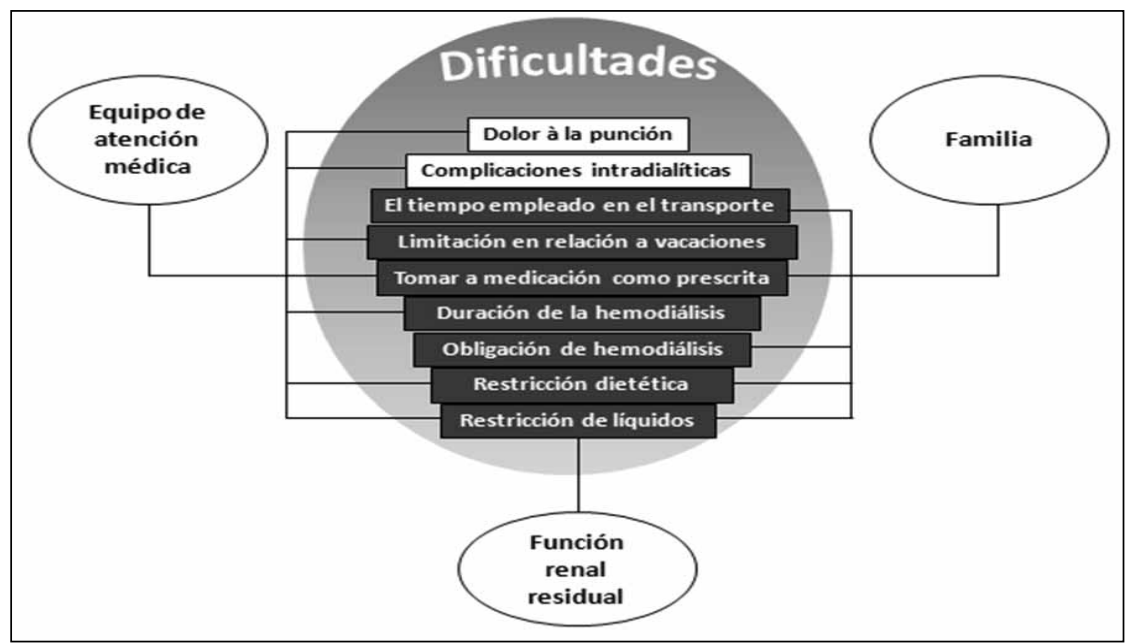

Figura 2. Relación entre las dificultades y los que influencian el manejo del régimen terapéutico. 
Los factores que complican la gestión de régimen terapéutico son: estar acostumbrado al consumo de agua, el calor del verano, fumar y tener que comer fuera. Los tres primeros factores dificultan la necesidad de restringir los líquidos. Fumar cigarrillos aumenta la sed y se puede modificar. Comer en los restaurantes, en los que no es posible proporcionar una dieta personalizada, afecta sobre todo a la necesidad de restringir ciertos alimentos de la dieta.

\section{Conclusiones}

Las dificultades más frecuentes fueron: la restricción de líquidos, la restricción dietética, cumplir con la hemodiálisis, la duración de la hemodiálisis, tomar la medicación prescrita, limitaciones en vacaciones, y dolor a la punción. Las dificultades, como cumplir con la HD, la duración de las sesiones, o toma de medicación, siguen siendo problemas importantes. Los principales factores que facilitan la gestión de régimen terapéutico fueron: el apoyo de los profesionales de la salud, el apoyo familiar, el mantenimiento de la función renal residual, rasgos de la personalidad, la actitud del equipo de salud y el apoyo de pacientes y amigos, tener un trabajo, estar en la lista de espera para trasplante renal, asociaciones de apoyo, y la habilidad de la enfermera. La familia parece tener particular importancia ayudando al paciente a cumplir con las sesiones de HD, a cumplir la dieta, la medicación y el transporte. Mantener la función renal residual permite mejor tolerancia a la restricción de líquidos. Los principales factores que dificultan la gestión del tratamiento son estar acostumbrado al consumo de líquidos, el calor del verano, el humo del tabaco, o tener que comer fuera. Estos factores afectan el control de los alimentos y los líquidos. Las enfermeras han de entender como los enfermos renales en HD administran el régimen de tratamiento, para que puedan ayudar y promover su autonomía. Es necesario estudiar la eficacia de las estrategias de adaptación para aconsejar a los pacientes en diálisis, en los esfuerzos para gestionar el régimen de tratamiento.

Recibido: 4 Octubre 2013

Revisado: 20 0ctubre 2013

Modificado: 29 Octubre 2013

Aceptado: 2 Noviembre 2013

\section{Bibliografía}

1. Sociedade Portuguesa de Nefrologia (SPN). Gabinete de Registo da Doença Renal Terminal. Relatório Anual 2013. [Acesso em: 07 Jul 2013]. Disponível em: http://www.spnefro.pt/comissoes_ gabinetes/Gabinete_registo_2012/registo_2012. pdf.

2. Bihl, M, Ferrans $C$, Powers M. Comparing stressors and quality of life of dialysis patients. American Nephrology Nurses Association. 1988; 15: 27-37.

3. Lok P. Stressors, coping mechanisms and quality of life among dialysis patients in Australia. Journal of Advanced Nursing, 1996; 23(5): 873-881.

4. Cristóvão F. Stress, coping and quality of life among chronic haemodialysis patients. "EDTNA/ERCA Journal". 1999; 25(4); 35-38. Inglês, Espanhol, Francês, Alemão, Italiano, Grego.

5. Mok E, Tam, B. Stressors and coping methods among chronic haemodialysis patients in Hong Kong. Journal of Clinical Nursing. 2001; 10(4); 503-511.

6. Welch JL, Austin, JK. Stressors, coping and depression in haemodialysis patients. Journal of Advance Nursing. 2001; 33(2): 200-7.

7. Kimmel PL. Depression in patients with chronic renal disease; What we know and what we need to know. Journal of Psychosomatic Research. 2002; 53(4); 951-956.

8. Tsay, SL., Lee, YC, e Lee, YC. Effects of an adaptation training programme for patients with endstage renal disease. Journal of Advance Nursing 2005; 50(1): 39-46.

9. Lazarus R, Folkman S. Stress, appraisal, and coping. New York: Springer Publishing Company, Inc., 1984. 445 p. ISBN 0-8261-4191-9.

10. Lindqvist $R$, Carlsson M, Sjodén PO. Coping strategies and quality of life among patients on hemodialysis and continuous ambulatory peritoneal dialysis. Scandinavian Journal of Caring Science. 1998; 12(4):223-30. 
11. Orem, DE. Nursing: Concepts of Practice. $6^{\text {th }} \mathrm{ed}$. St. Louis: Mosby; 2001. 542 p. ISBN 0-32-300864-X.

12. Torney AM, Alligood MR. Teóricas de Enfermagem e a sua obra. $5^{\mathrm{a}}$ ed. Loures: Lusociência; 2004.750 p., ISBN 972-8383-74-6.

13. Machado, MM. Adesão ao regime terapêutico - representações das pessoas com IRC sobre 0 contributo dos enfermeiros [Tese de mestrado]. Universidade do Minho - Instituto de Educação e Psicologia; 2009. 272 p.
14. Gurklis JA, Menke E. Identification of stressors and use of coping methods in chronic hemodialysis patients. Nursing Research, 37(4) Jul-Aug 1988 p. 236-239,248.

15. Curtin RB, Mapes DL. Health care management strategies of long-term dialysis survivors. Nephrology Nursing Journal, 2001, 28(4): 385-92; 393-4.

16. Daugirdas JT, Blake PG, Ing TS. Manual de Diálise. $4^{a}$ ed. Rio de Janeiro: Guanabara Koogan; 2010. 712 p. ISBN 978-85-277-1431-0. 